\title{
XXVI.
}

\section{Ein Saugpolster in der menschlichen Backe.}

\author{
Von Dr. H. Ranke, \\ Professor der Kinderheilkunde in München.
}

(Hierzu Taf. XVIII-XIX.)

Bei Kindern, welche im Laufe des ersten Lebensjahres in Folge von Diarrhöen der Atrophie verfallen, bemerkt man hänfig, wenn sie schon den grössten Theil ihres im Unterhautbindegewebe abgelagerten Fettes verloren haben, in beiden Backen, in der Umgebung des Ductus Stenonianus, eine kleine Anschwellung, etwa von der Grösse einer gespaltenen Haselnuss. Diese Anschwellung ist ziemlich rundlich abgegrenzt, fühlt sich weich an und kann, wenn sie durch den betastenden Finger zwischen beiden Kiefern hin und her bewegt wird, für den Ungeübten Fluctuation vortäuschen.

Ich hatte diesen scheinbaren Tumor, der sich zaweilen wie eine Cyste in der Backe darstellt, vor Jahren offers untersucht und gefunden, dass er aus einer, in eine bindegewebige Umhüllung eingeschlossenen Partie Fett besteht.

Dieser Befund erschien mir auffallend; denn anfangs konnte ich durchans nicht einsehen, warum selbst bei stärkster allgemeiner Abmagerung diese Partie Fett in der Backe meist nicht resorbirt werden sollte.

Allerdings in einzelnen Fällen fand ich auch sie grösstentheils resorbirt, doch bildeten derartige Fälle die Ausnahme.

Im Laufe der Jahre habe ich nun häufig in meiner Poliklinik, in welcher ich leider sehr oft Gelegenheit habe, junge Kinder, welche in Folge von Breinahrung und dem Gebrauch des Schnullers atrophisch geworden, vorzustellen, über das Eigenthümliche der Nichtresorption dieser Partie Backenfett gesprochen und betont, dass für diese Erscheinung ein besonderer physio- 
logischer Grund vorliegen müsse, den ich bis jetzt nicht zu enträthseln vermöge.

1881 stellte ich, angeregt durch die Versuche von $V_{0} i^{1}{ }^{2}$, Chossat und Anderen an hungernden Thieren, einem meiner Schüler, Herrn Dr. Wilhelm Ohlmüller, die Aufgabe, über die Abnahme der einzelnen Organe bei an Atrophie gestorbenen Kindern exacte Untersuchungen anzustellen.

Ohlmüller lieferte eine verdienstliche Arbeit, die er unter gütiger Leitung v. Voit's in dessen physiologischem Institut ausgeführt hat $\left.{ }^{2}\right)$. Er fand, was ich schon als Vermuthung ausgesprochen hatte, dass die Abnahme der einzelnen Organe bei in Folge von Darmkatarh atrophirenden Kindern in ganz ähnlichem Verhältniss vor sich geht, wie bei verhungernden Thieren. Insbesondere wies er nach, dass die Centralorgane des Nervensystems und der Circulation, Gehirn und Herz, bei atrophischen Kindern wie bei verhungernden Thieren eine nur geringe Abnahme zeigen, während von dem Fett im Unterhautbindegewebe bis zu 97 pCt. zur Resorption gelangen können. Zu dem Gesammtverluste des Körpers an Fett trugen in einem der von 0 hlmüller untersuchten Fälle Haut. und Unterhautbindegewebe 91 pCt., die Muskeln 5 pCt., die Knochen 2 pCt. bei; der Verlust aller übrigen Organe an Fett betrug nur 2 p(t. $\left.{ }^{3}\right)$.

Durch die Ohlmüller'sche Arbeit wurde die Nichtresorption des Backenfettes in der Umgebung des Duct. Stenonianus noch räthselhafter. Wenn beim atrophirenden Kinde die Haut und das unter derselben liegende Bindegewebe 97 pCt. ihres ursprünglichen Fettgehaltes verlieren können, was kann der Grund sein, dass diese umschriebene Partie Fett in der Backe der Resorption widersteht?

Bei einer Discussion über die Ohlmüller'sche Arbeit in der Münchener morphologisch-physiologischen Gesellschaft gab ich dieser Frage Ausdruck, worauf Herr Prof. Kupffer erwiderte,

1) Voit, in Hermann's Handb. d. Pbysiol. Bd.6.

3) Wilhelm Ohlmüller, Ueber die Abnabme der einzelnen Organe bei an Atrophie gestorbenen Kindern. Zeitschr. f. Biologie XVIII. Bd. 1882. S. 78 .

3) a. a. 0. S. 99 u. 1.00 . 
dass er sich aus seiner Studienzeit an eine unter der Aegide des damaligen Professors der Geburtshülfe in Dorpat, Walter, erschienene Dissertation erinnere, in welcher Fettkugeln in der Wange anatomisch beschrieben wurden.

Nach längerem Suchen gelang es in der That, diese im Jahre $1853 \mathrm{zu}$ Dorpat erschienene und seitdem von den Anatomen sowohl als den Pädiatrikern, wie es scheint, fast gänzlich unbeachtet gebliebene Dissertation aufzufinden.

Dieselbe ist betitelt: "De corpusculo quodam adiposo in hominum genis obvio. Scripsit Wilfried Gehewe. Dorpati Livonorum MDCCCLIII." und mit einer Abbildung versehen.

Zunächst ist aus dieser Abhandlung hervorzuheben, dass nicht Gehewe das Corpusculum adiposum der Wange zuerst auffand, sondern sein Lehrer Prof. Walter, welcher dasselbe anfangs der dreissiger Jahre zufällig bei einem Neugebornen beobachtete, dessen Hinterhaupt in viele Stücke zerschmettert worden war. In diesem Falle waren nehmlich die Körperchen beiderseits durch Schleimhautwunden in die Mundhöhle gelangt und lagen auf dem Rücken der Zunge, je an einem Stiele haftend, welch letzterer sich weit in die Wange verfolgen liess.

Walter habe anfangs nicht gewusst, was er von diesen Körperchen ("Corpuscula rotunda, diametro linearum 3-4 praedita, laevia, fere splendentia, satis spissa ac solida"), über welche er in den anatomischen Handbüchem keine Aufklärung fand, halten sollte, bis er sich durch zahlreiche Sectionen an Neugebornen von dem regelmässigen Vorkommen derselben überzeugte: Er habe seitdem das Körperchen bei Sectionen stets demonstrirt und pflege dasselbe auch in seinen Vorlesungen über Kinderkrankheiten zu erwähnen ${ }^{1}$ ).

Gehewe beginnt seine Arbeit damit, dass er nachweist, dass bereits Winslow, Bichat, Malgaigne, Krause, Nuhn in ibren Werken kurze Andeutungen über das Körperchen gegeben haben. Ich übergehe hier diesen historischen Nachweis und verweise auf den Anhang, in welchem ich die wichtigsten Stellen der einschlägigen Literatur aus älterer und neuerer Zeit zusammengestellt habe.

) a. a. 0. S. 22 น. 23.

Archiv f. pathol. Anat. Bd. XCVII. Het. 3 . 
Gehew 0 theilt seine Arbeit in drei Capitel, deren erstes der anatomischen Beschreibung des Körperchens gewidmet ist. Diesem Capitel ist in der Haupsache Folgendes zu entnehmen:

Wenn man über die Wange eines Neugebornen einen geraden Schnitt führt vom Mundwinkel bis zur Incisura intertragica des Ohres, so findet man, etwa in der Mitte dieses Schnittes, unter dem Panniculus adiposus einen von einer dünen Fascie umgebenen Fettkörper von rundlicher oder auch länglicher Gestalt, an Grösse und Form einer Haselnuss oder Mandel ähnlich. Seine Grösse varïrt etwas und beträgt durchschnittlich etwa $\frac{3}{4}$ Zoll $(2 \mathrm{~cm})$ in der Länge und je $\frac{1}{2}$ Zoll $(1,5 \mathrm{~cm})$ in Breite und Dicke. Das Körperchen ist häufig gelappt, zuweilen zeigt es jedoch eine vollkommen glatte Oberfläche. Der Ductus Stenonianus verläuft entweder gerade darüber hinweg oder in einer Spalte zwischen zwei Lappen desselben.

Auf der Seite, die dem M. buccinator zugewendet ist, hat das Körperchen einen Stiel, der durch eine Art Kanal nahe unter dem Os zygomaticum durchgeht und sich in die Fossa sphenopalatina inserirt. Dieser Stiel hat einen kleinen Ast, der mit dem M. temporalis unter dem Jochbogen zur Fossa temporalis aufsteigt und den oberen Rand des Arcus zygomaticus um einige Linien überragt.

Das Körperchen persistirt durch alle Lebensalter und wurde noch bei einer abgemagerten Frau von 60 Jahren gefunden. Bei Erwachsenen ist jedoch seine Gestalt mehr zusammengedrückt und abgeplattet, auch liegt es nicht mehr am vorderen Rande des Masseter, sondern hat sich mit seinem Stiel mehr zurückgezogen und tritt überhaupt weniger hervor. Der Stiel ist dicker geworden, doch ist die Grösse des Körperchens bei fetten wie mageren Menschen ungefähr die gleiche.

Die Structur des Körperchens wird als homogen beschrieben; es bestehe hauptsächlich aus Fettzellen und werde von nur wenigen und kleinen Blutgefässen durchzogen.

Im zweiten Capitel theilt Gehewe dann zwei Fälle mit, welche die Bedeutung des Körperchens für die gerichtliche Medicin darthun sollen, was der Autor als das wesentlichste Ergebniss seiner Untersuchung zu halten scheint ${ }^{1}$ ).

i) a. a. O. S.1. 
Der erste Fall, von Prof. Walter beobachtet, wurde bereits oben kurz skizzirt, ein zweiter ähnlicher Fall, bei welchem jedoch die nähere Natur des Körperchens nicht erkannt wurde, was zu verschiedenen gutachtlichen Aeusserungen Veranlassung gab, findet sich ausführlich beschrieben in der deutschen Zeitschrift für die Staatsarzneikunde von Schneider und Schürmayer, 1852, neue Folge, Bd. XI, S. 66.

Das dritte und Schluss-Capitel bringt noch verschiedenartige Beobachtungen besonders auch über die Entwickelung des Körperchens und einige Bemerkungen über die physiologische Bedeutung desselben.

Bei mageren Kindern ist das Körperchen zwar kleiner als bei fetten, es tritt aber bei ersteren deutlicher hervor, weil das granulirte Fett, das es sonst umgiebt, in geringerer Menge vorhanden ist.

Die Grösse des Körperchens ist nicht immer in beiden Wangen die gleiche, auch seine Gestalt variirt ziemlich bedeutend. Zuweilen hat es, wie bereits erwähnt, eine vollkommen glatte Oberfläche, zuweilen zerfällt es in zwei, seltener in mehrere Lappen und Läppchen und kann in letzterem Fall die Gestalt einer Maulbeere annehmen; zuweilen ist es in der einen Wange gelappt, in der anderen nicht; auch kann es vorkommen, dass der Duct. Stenonianus auf der einen Seite über das Körperchen hinwegzieht, während er sich auf der anderen Seite, etwa noch mit einer kleinen Parotis accessoria versehen, zwischen die Lappen desselben einsenkt.

Beim Fötus finden sich die ersten, wenn auch noch etwas undeutlichen Spuren des Körperchens bereits im 3. Monat, während es im 4. Monat schon deutlich als rundliches Knötchen von Stecknadelkopfgrösse hervortritt. Im 5. Monat hat es Grösse und Gestalt einer Linse, im 6. die einer Erbse u. s. w.

Bei Thieren fand Gehewe das Körperchen nicht, wenigstens nicht an der Stelle, die es beim Menschen einnimmt.

Zum Schluss wirft Gehewe die Frage auf nach der Function des Körperchens und kommt zu dem Resultat, diese Functionen müssten andere sein als die des Fettes im Allgemeinen, vielleicht lägen sie darin, dass es auf mechanischem Wege den Saugact unterstïtze; denn das Körperchen sei beim Neugebornen 
vollkommen entwickelt, während der M. buccinator noch so schwach sei, dass er beim Saugen zwischen die Kiefer gezogen werden müsse, wenn der Wange nicht durch das Körperchen der nöthige Halt verliehen wurde.

Soweit Gehewe.

Seine Arbeit, welcher eine die Flächenansicht des Körperchens darstellende Abbildung beigegeben ist, enthält offenbar viele neue Thatsachen, genügt aber doch wohl nicht, um die Bedeutung des Gegenstandes, den sie behandelt, endgültig festzustellen. Wir erhalten durch sie eine werthvolle anatomische Schilderung eines bis dahin kaum beachteten Gebildes, ausserdem aber nur Andeutungen und Vermuthungen äber die mögliche Function desselben.

Gerade das Unbestimmte der Resultate Gehewe's in letzterer Richtung dürfte die Ursache gewesen sein, warum seine sonst so verdienstvolle Arbeit in der Wissenschaft so wenig Beachtung gefunden hat.

Die Sache scheint mir aber auf Grund eigener Beobachtungen, welche diejenigen Gehewe's vervollständigen und ergänzen, so gelagert zu sein, dass ich glaube, mit vollster Entschiedenheit aussprechen zu können:

1. Das fragliche Körperchen ist ein selbständiges anatomisches Gebilde.

2. Seine biologische Bedeutung ist die eines Saugpolsters, das heisst, es dient als Widerlager des Buccinators zur Unterstützung der Function der Backe beim Saugact.

Ich ersuche nun den geehrten Leser die Tafel der Abbildungen in's Auge zu fassen (s. auch die Erklärung der Abbildungen $\mathrm{S} .546 \mathrm{u}$. f.).

Fig. 1 zeigt ein grobknochiges, atrophisches, 5 Monate altes Kind, dessen Panniculus adiposus in Folge langandauernder Diarrhöen zum grossen Theil geschwunden ist, wie die Falten am Halse und das Hervortreten der Rippen erkennen lassen. Das Fettpolster der Backe ist dagegen wohl erhalten und tritt, besonders beim Schreien in sehr markirter Weise hervor.

Das Bestehenbleiben dieser umschriebenen Fottansammlung in der Backe bei hochgradig abgemagerten, atrophischen Kindern war es, was, wie eingangs erwähnt, zuerst meine Aufmerksam- 
keit anf den Gegenstand lenkte, besonders nachdem. ich weiterhin gefunden hatte, dass dieses Fett stets in eine besondere, bindegewebige Umhüllung eingeschlossen ist.

Schon aus dieser Beobachtung geht hervor, dass es sich hier um ein Gebilde von einer gewissen Selbständigkeit und besonderen Function handeln müsse.

Fig. 2 zeigt das Saugpolster eines Neugebornen in seiner natürlichen Lage von aussen und in seinen Verhältnissen zur Umgebung.

Das Polster stellt sich als ein von dem Panniculus adiposus der Wange scharf sich abhebender, in eine Bindegewebskapsel eingeschlossener, selbständiger Körper dar. In diesem Falle ist es zweilappig und der Duct. Stenonianus verläuft in der durch die beideñ Lappen gebildeten Rinne. Nach oben und vorn ist der Stiel des Polsters erkennbar.

In Fig. 3 ist der Stiel, an welchem das Polster aufgehängt ist, dargestellt. Der Stiel inserirt sich in die Fossa sphenopalatina. Um denselben in seinem ganzen Verlaufe darzustellen, wurde der Jochbogen mit dem Masseter nach unten, der aufsteigende Unterkieferast sammt Gelenkkopf und dem Temporalis nach oben geschlagen.

Wie das Polster selbst, so ist anch sein Stiel in seinem ganzen Verlaufe von einer eigenen bindegewebigen Hülle umgeben und lässt sich von dem übrigen vorhandenen Fett leicht vollständig trennen.

Durch diesen Stiel gewinnt das Saugpolster die Fähigkeit allen Bewegungen der Backe leicht zu folgen, ohne doch jemals aus seiner Lage kommen zu können.

Fig. 4 stellt einen Horizontalschnitt durch Backen und Mundhöhle eines Neugebornen dar.

Das Polster hebt sich von dem übrigen Wangenfett scharf ab und lässt sein Verhältniss zum Buccinator deutlich erkennen. Ohne jede $Z$ wischensubstanz liegt es unmittelbar einem Theile dieses dünnen Muskels auf und bildet so ein Widerlager desselben, welches durch seine theilweise Auflagerung auf den Masseter, besonders aber durch die feste Insertion des Polsterstieles an Wirkung gewinnt.

Fig. 5 zeigt dieselben Verhältnisse bei schwacher Vergrössee- 
rung. Die Zeichnung stellt einen Horizontalschnitt des blau injicirten, mit Carmin imbibirten P'olsters sammt Buccinator und Wangenschleimhaut dar.

Bekanntlich sind Backenschleimhaut und Buccinator ohne jede Zwischensubstanz innig mit einander verbunden. Dieses Verhältniss tritt in der Zeichnung deutlich hervor, aber auch das Sangpolster ist an einer Stelle mit dem Buccinator durch bindegewebige Züge innig verbunden.

Seine Kapsel ist scharf begrenzt. Von ihr aus gehen Lamellen in das Innere und treten hier in gegenseitige Verbindung, so dass an den Durchschnitten ein Balkennetz auftritt. Die injicirten Gefässe zeigen, dass das Polster mit Blut reichlich versorgt wird.

Die dem Polster nach oben und unten angrenzende Bindesubstanz mit eingelagerten Fettpartien unterscheidet sich scharf von dem Gewebe des Polsters und zeigt einen wesentlich verschiedenen Bau.

Von Interesse ist der zapfenförmige Vorsprung, welchen Schleimhaut und Buccinator an der Stelle bilden, wo das Polster mit diesem Muskel verbunden ist. Derselbe Vorsprung zeigt sich auch bei Fig. 7 .

Fig. 6 stellt einen kleinen Ausschnitt aus der Peripherie des Polsters unter nicht ganz hundertfacher Vergrösserung dar. Die Kapsel ist stark entwickelt und aus Bindegewebe gebildet, von welchem Lamellen nach dem Inneren des Polsters abgehen und den ausserdern nur aus Fettzellen bestehenden Inhalt desselben in grössere und kleinere Unterabtheilungen gliedern.

Grössere Gefässstämme verlaufen in der Kapsel und senken sich von da in das Innere, dessen sämmtliche Fettzellen ein reiches Capillarnetz umspinnt.

Ein besonders instructives Präparat dürfte endlich in Fig. 7 dargestellt sein.

Dasselbe stellt einen siebenfach vergrösserten Frontalschnitt des Kopfes eines ca. 4 Monate alten Fötus dar. Der Schnitt stammt aus einer Collection frontaler Durchschnitte des Kopfes, welche Herr Prof. Rüdinger schon vor längerer Zeit hergestellt hat und mir freundlichst zur Benutzung überliess, wofür ich ihm an dieser Stelle besten Dank sage. 
Das Polster ist schon als deutlich ausgeprägter Körper vorhanden, welcher sich von dem Panniculus adiposus der Umgebung scharf abgrenzt. Eine bindegewebige Kapsel umgiebt dasselbe und ein dicker, breiter Faserzug verbindet es mit dem Buccinator, welchem es in dieser Periode der Entwickelung noch nicht so enge anliegt wie in späterer Zeit. Unter der Verbindungsstelle zwischen. Polster und Buccinator bildet dieser Muskel mit der Mundschleimhaut einen ganz ähnlichen, zapfenförmigen, mit verschiedenen Einkerbungen versehenen Vorsprung wie in Fig. 5.

Von Interesse ist auch die theils von dem M. subcutaneus colli, theils von dem Risorius abstammende Muskellage, welche das Polster von aussen, theilweise auch von oben und unten, begrenzt.

Ich glaube die Sprache dieser Abbildungen ist so allgemeinverständlich, dass ich denselben nur noch wenige Worte beizufügen habe.

Die Gehewe'sche Ansicht, dass das Polster nur von wenigen und kleinen Blutgefässen versorgt werde, erweist sich auf Grund der Abbildungen No.5 und 6 als irrig. Im Gegentheil ist die Blutversorgung eine reichliche und betheiligen sich an derselben verschiedene Arterien, nehmlich die Art. maxillaris externa, transversa faciei und maxillaris interna.

Ueber die Form des Polsters bei Erwachsenen wurde eine Abbildung nicht beigegeben, weil eine solche bereits existirt, nehmlich von P. Tillaux, Traité d'Anatomie Topographique, Deuxième Edition, Paris 1879, p. 287, Fig. 96. Diese Figur stellt einen Horizontalschnitt der Backe einer erwachsenen Frau dar, in welchem die "boule graisseuse de Bichat" deutlich hervortritt. Letztere ist aber nichts Anderes als das Saugpolster ${ }^{1}$ ). Indem ich auf die genannte Abbildung verweise, mache ich besonders auf das dort hervortretende, wesentlich veränderte Verhalten des Polsters zum Masseter aufmerksam. Dasselbe hat sich beim Erwachsenen mit seinem Stiel etwas verkürzt und liegt nun unter dem genannten Muskel, während es beim Säugling den vorderen Rand desselben theilweise bedeckt.

Der Grund, warum das Polster, wie es scheint, bei Thieren fehlt, dürfte in der stärkeren Entwicklung der Musculatur bei denselben und dem Mangel einer eigentlichen Backe seine Erklärung

1) S. Anbang S. 539, 
finden. Leider hatte ioh keine Gelegenheit Affen untersuchen zu können; es würde aber von Interesse sein bei anthropoiden Affen das Vorkommen oder Fehlen des Polsters zu constatiren.

Dass das Polster durch seine eigenthümliche Bildung und Lagerung für das Verständniss der Lipome in der Backengegend von Wichtigkeit sein wird, bedarf kaum der Erwähnung. In der That macht schon Virchow in seinem Buche über "die krank-. haften Geschwülste", Berlin 1863, darauf aufmerksam und erwähnt dabei, wie ich nachträglich finde, in einer Anmerkung sogar die Gehewe'sche Dissertation (s. Anhang S.542). Weitere Andeutungen darüber finden sich in dem genannten Tillaux'schen Werke (s. Anhang S. 543 u.f.).

Zum Schluss nur noch wenige Bemerkungen.

Ich glaube in Vorstehendem den Beweis erbracht zu haben, dass das fragliche Fettpolster ein selbständiges, anatomisches Gebilde ist.

Aber auch der Beweis, dass es ein Saugpolster (Tomentum suctus buccale) ist, das heisst, dass es zur Unterstützung der Function der Backe beim Saugen dient, dürfte sich schon aus den bisherigen Schilderungen ergeben haben.

Dass durch die Anheftung des Polsters an den Buccinator die Function dieses Muskels eine Beeinflussung in der Weise erfahren muss, dass derselbe bei den Saugbewegungen nicht zu weit zwischen die Kiefer gezogen werden kann, ist selbstverständlich, besonders wenn man bedenkt, dass das Polster durch seinen Stiel in der Fossa sphenopalatina fest fixirt ist.

Von besonderer Wichtigkeit erscheint aber ausserdem die Thatsache, dass das Polster beim Neugebornen mindestens schon die gleiche Grösse hat, oder selbst grösser ist, als beim Erwachsenen und jedenfalls bei ersterem viel deutlicher hervortritt. Diese Thatsache lässt keine andere Erklärung zu, als dass das Polster eine für das Säuglingsalter besonders wichtige Function haben müsse. Diese Function kann aber nach Lage der anatomischen Verhältnisse eben nur auf den Saugact Bezug haben.

Als ein Analogon hiezu erinnere ich an die bekannte Thatsache, dass der M. labii proprius, der Saugmuskel der Lippe, beim Säugling relativ wesentlich stärker ausgebildet ist als beim Erwachsenen, und dass die innere Zone der rothen Lippensohleim- 
haut im Säuglingsalter weiche, bis $1 \mathrm{~mm}$ lange, zottenähnliche Hervorragungen besitzt, welche offenbar zum besseren Ansaugen an die Brustwarze dienen und später sich grossentheils zurückbilden (W. Krause) ${ }^{1}$ ).

Dass das Polster bei der Atrophie der Kinder der Fettresorption zu widerstehen pflegt, hat wohl darin seinen Grund, dass es gewöhnlich bis zum Tode in fast beständiger Function erbalten und deshalb reichlicher als andere Organe mit Blut versorgt wird.

Gerade mutterlos aufgezogene Kinder, die ja weitaus das grösste Contingent der Atrophie liefern, machen an dem Schnuller oder Sauggummi, den ihnen unverständige Pflegerinnen in den Mund geben, fast ununterbrochene Saugbewegungen.

Wir wissen aber aus den Versuchen verschiedener Forscher an hungernden Thieren und aus Ohlmüller's ${ }^{2}$ ) Beobachtungen an atrophischen Kindern, dass thätige Organe im Allgemeinen eine Stoffabnahme nicht zeigen, weil jedes innerhalb der Grenzen seiner physiologischen Leistungsfähigkeit stärker thätige Organ entsprechend stärker ernährt wird.

\section{A $n$ h a $n g$.}

Uebersicht über die wichtigsten bisher in der Literatur vorhandenen auf das Saugpolster bezüglichen resp. zu beziehenden Andeutungen.

Die erste auf das Saugpolster zu beziehende Bemerkung findet sich bei Heister, welcher dasselbe für eine Drüse hielt und als Glandula molaris beschrieb und abbildete.

Es heisst nehmlich in Dr. Laurentius Heisteri Compendium anatom. Edit. IV, Norimbergae 1732, Tom. I, p. 146:

"Glandulae duae, utrinque una in eodem cadavere in genis circa dentes molares posteriores maxillae superioris, aliquot osculis excretoriis, in os hiantibus a me observatae sunt. Vid. Not. 57 et Tab. VIII. Fig. 36 et $37 . "$

1) Handb. d. menschl. Anatomie von C. Fr. Th. Krause, dritte von W. Krause neubearbeitete Auflage. Hannover 1879. S. 387.

9) a. a. O. S. 103 . 
Figur 36 stellt einen maulbeerförmigen, gelappten, $19 \mathrm{~mm}$ langen and $13 \mathrm{~mm}$ breiten Körper dar, während Figur 37 einige kleine Oeffnungen der Mundschleimhaut in der Gegend des letzten Backenzahnes erkennen lässt.

Not. 57, auf welche oben hingewiesen wird, findet sich in Tom. II p. 109 und lautet: "In hoc ipso subjecto duas insignes glandulas conglomeratas, utrinque unam circa dentes molares posteriores maxillae superioris, inter musculum buccinatorem et masseterem, ea magnitudine et figura ut in fig. 36 delineantur, deprehendi, quae ob situm molares dici possent: in quarum qualibet duo magna oscula, punctis lacrymalibus duplo ampliora, quae facillime setas crassas, itemque stylos quibus in fistula lacrymali utor, ad duarum linearum profundidatem, admittebant, perbelle conspicebantur; in sinistra vero adhuo duo vel tria paullo minora foraminula aderant, quae omnia membranam oris et buccinatorem musculum penetrabant, glandulaque leviter pressa guttulam quodlibet salivae pellucidae dabant. Fig. 36 ostendit faciem externam glandulae sinistri lateris ex pluribus minoribus, fere ut mori fructus compactam: unde quod ad compositas sive conglomeratas, ut vocant, pertineat, cognosci potest; quae vero in hoo cadavere, ubi dentes posteriores deficiebant, cum glandalosa palati substantia cohaerebat. An hae glandulae semper adsint, earumque ductus sive oscula excretoria in aliis quoque adeo sint manifesta, affirmare non audeo, sed ulterius inquirendum est."

Ich halte es für zweifellos, dass der von Heister hier abgebildete Körper das Saugpolster darstellt, welches in diesem Falle durch seinen gelappten, maulbeerartigen Bau, wie solcher nach S. 9 ja öfters beobachtet wird, um so leichter den Irrthum, dass es sich um eine Drüse handle, aufkommen liess, als mehrere kleine Oeffnungen in der Richtung gegen das Polster zu führen schienen.

Nun liegt aber in nächster Nähe des Polsters, hinter der Ausmündung des Duct. Stenonianus und unmittelbar über dem Buccinator eine Gruppe hanfkorngrosser Speicheldrüsen (Glandulae buccales s. molares), deren kurze Ausführungsgänge, welche in diesem Falle vielleicht etwas erweitert waren, Heister irrthümlicher Weise mit dem Polster in Beziehung brachte.

Die topographischen Verhältnisse des Saugpolsters und der 
Molardrüsen finden sich klar dargestellt in dem bereits erwähnten Horizontalschnitt der Backe eines Erwachsenen bei J. Tillaux, Traité d'Anatomie topographique, II. éditon, Paris 1879, p. 287, Fig. 96, worauf ioh hiermit wiederholt verweise.

Winslow (Jac. Beningn. Winslow, Expositio anatomica structurae corporis humani, Francofurti et Lipsiae 1753, Tom. IV) steht ganz unter dem Einfluss des Heister'schen Irrthums, sagt jedoch, man könne leicht die beiden Drüsen für $z$ wei besondere Fettknollen halten.

Unter der Ueberschrift Glandulae Salivales beschreibt er nehmlich p. $264 \$ 583$ die Heister'schen Glandulae molares folgendermaassen: "Duae Glandulae ejusdem fere speciei quam praecedentes (glandulae sublinguales) sunt, quarum quaelibet in latere suo inter Musculum Masseterem atque Musculum Buccinatorem sita est. Facile in quibusdam subjectis pro duobus peculiaribus pinguedinis Glomeribus haberentur. Parvos Tubos producunt, qui Buccinatorem perforant, atque in Oris cavitatem e regione circiter uItimorum Dentium Molatium hiant. Id quod Dominum Heisterum, qui easdem primus luci exposuit, ut easdem Glandulas Molares vocaverit, impulit."

Zu einer richtigeren Auffassung über die Natur des fraglichen Körpers gelangte am Anfang des Jahrhunderts Bichat ${ }^{1}$ ), welcher sich in seiner Allgemeinen Anatomie im Jahre 1801 folgendermaassen über denselben äussert: "La graisse est ramassée, chez le foetus, en petits globules plus ou moins arrondis, ce qui donne à son ensemble un aspect granulé. Souvent même il se fait des amas assez considérables: par exemple, il y a presque toujours à cette époque, entre le buccinateur, le masseter et les tegumens, une espèce de boule graisseuse qui fait un corps isolé de la graisse environnante, etqu'on extraiten totalité: elle contribue beaucoup à la saillie remarquable que les joues font à cette époque de la vie."

Diese Bemerkung veranlasste die frauzösischen Anatomen seitdem das Polster als "la boule graisseuse de Bichat" zu

I) Xavier Bichat, Anatomie générale. Nouvelle Édition. Tome I. Paris 1830. p. 79 . 
bezeichnen, was zur Folge hatte, dass in Frankreich von demselben wesentlich mehr Erwähnung geschah als in Deutschland.

J. F. Malgaigne ${ }^{1}$ ) äussert sich im Jahre 1830 bei Aufzählung der verschiedenen Lagen der Magengegend, „region génienne" folgendermaassen:

$\ldots 3^{\circ}$. "En haut et en dehors, les muscles petit et grand zygomatiques; en bas le peaucier et le triangulaire; entre ces muscles, et près du masséter, un peloton adipeux spécial, qui chez les enfants et les sujets chargés d'embonpoint constitue la saillie arrondie de la joue, et dont l'absence laisse un creux remarquable chez les personnes maigres."

Auf p. 763 erwähnt Malgaigne auch den Stiel des Polsters "Le tissue adipeux prédomine surtout à la réunion de la région génienne avec la région massétérine. Là se trouve un peloton adipeux tout spécial, qui, chez les enfants où il est très-devéloppé, fait quelquefois hernie entre les bords des plaies siégeant en cet endroit; s'il tient au fond de la plaie par un pédicule assez gros pour continuer à vivre, on peut le remettre en place avant de proceder à la reunion" ....

Die französischen Autoren zoigen in dieser Beziehung eine sehr viel eingehendere Kenntniss der Verhältnisse der Backe als die Verfasser der deutschen anatomischen Lehrbücher der damaligen Zeit.

So spricht C. F. T. Krause ${ }^{2}$ ) im Jahre 1841 nur von einer "ansehnlichen. Nenge Fettgewebe" über dem Buccinator und Fr. Arnold ${ }^{3}$ ) beschränkt sich (im J. 1847) auf die Bemerkung: "Die äussere Hautschicht . . besitzt an den Lippen eine körnige und ziemlich dünne, an den Backen aber eine ansehnliche, bei fetten Individuen und besonders bei Kindern sehr dicke Fettlage. "

Etwas ausführlicher äussert sich $\mathrm{A}$. $\mathrm{Nuhn}^{4}$ ), indem er er-

3) J. F. Malgaigne, Traité d'anatomie chirurgicale. Bruxelles 1830. Tome I. p. 795. (Deuxième Édition. Paris 1859. Tome I. p. 751.)

2) O. Fr. Th. Krause, Handb. d. menschl. Anatomie. Hannover 1841. II. Aufl. S. 308.

3) Fr. Arnold, Handb. d. Anatomie d. Menschen. Freiburg i. B. 1847. II. Bd. S. 41.

4) Anton Nuhn, Handb. d. spec. chirurg. Anatomie. Mannheim 1845. I. Bà. S. 188. 
wähnt, dass sich „Fett in grosser Menge an der Backe, auf den M. buccinator bis zum Rande des masseter, angesammelt findet, wo es bei grösserer Anhäufung die Bauschbacke erzeugt, dagegen, wenn es ungewöhnlich vermindert wird, wie z. B. bei schlechter Ernährung, auszehrenden Krankheiten und dergleichen, sein Mangel die eingefallene Wange bedingt u.s. w."

Soweit die bemerkenswerthesten Angaben über das Polster in der Literatur bis zum Erscheinen der Gehewe'schen Arbeit im Jahre 1853.

Dass die Gehewe'sche Schrift, trotz der vielen neuen Thatsachen die sie enthielt, bei den Kinderärzten sowohl als bei den Anatomen fast ganz unbeachtet blieb, wurde bereits oben erwähnt.

Im Jahre 1853 machte auch $\mathrm{Hyrtl}$ in seinem Lehrbuch der topographischen Anatomie, Wien 1853, S. 208 von dem Polster Erwähnung. Es heisst dort: „Am vorderen Rande des Masseters bildet das Unterhautzellgewebe einen den Duct. Stenonianus einhüllenden Klumpen, der sich tief in die Kluft zwischen den Kronenfortsatz des Unterkiefers und den Oberkieferkörper einsenkt und durch die Reibung, die er bei den Bewegungen der Kauorgane erfährt, so geglättet wird, dass er mit einer serösen Haut, einer Art Bursa mucosa, überzogen wird."

Dass diese Bemerkung Hyrtl's nicht durch die Gehewe'sche Arbeit beeinflusst wurde, ist klar, denn da Gehewe gezeigt hatte, dass das Polster bei Neugebornen vollkommen entwickelt ist, hätte Hyrtl unmöglich auf den Irrthum verfallen können, dass die glättende Wirkung der Bewegungen der Kauorgane dazu gehöre, um die häutige Umbüllung des Polsters zur Entwicklung zu bringen.

Hyrtl kannte dagegen Bichat's Angaben, wie der nachfolgende Passus aus seinem Lehrbuch d. Anat. d. Menschen, 8. Aufl., Wien 1863 S. 395 beweist. Es heisst dort: „Zwischen beiden Blättern der Fascia buccalis bleibt am vorderen Rande des Masseter ein Raum übrig, welcher durch einen rundlichen Fettknollen ausgefüllt wird. Diese Fettmasse, von Bichat la boule graisseuse de la joue genannt, dringt zwischen der Aussenfläche des Buccinator und der Innenfläche des Unterkieferastes bis in die Fossa temporalis hinauf. Schwindet sie bei allgemeiner 
Abmagerung, so fält die Backenhaut zo einer Grube ein und bildet die den abgezehrten Gesichtern eigenthümliche hohle Wange."

Der einzige deutsche Autor, bei welchem ich die Gehewe'sche Arbeit citirt finde, ist, wie bereits angegeben, Rudolf Virchow, „Die krankhaften Geschwülste", Berlin 1863.

Derselbe schreibt a. a. 0. S. 395: „Schliesslich will ich, ankniipfend an ein Präparat unserer Sammlung (Präparat No. 122 vom Jahre 1861), noch den besonderen Fall hervorheben, der öfters zu Verwechselungen Veranlassung giebt, dass sich nehmlich aus einem bestimmten Fettgebilde, das nicht besonders beschrieben zu werden pflegt, ein solches Gewächs (Lipom) entwickelt. Es giebt in der Wange eine besondere Fettmasse, welche beim Erwachsenen in der Regel weniger hervortritt, als bei Kindern, namentlich neugebornen, eine Fettmasse, welche von der Fossa canina sich wie gestielt in die Dicke der Wange fortsetzt und welche sehon Heister (1741) u. A. beschrieben haben, welehe aber immer wieder vergessen worden ist. Sie trägt den Namen des Fettkörpers der Wange, Corpus adiposum malae (Gehewe, De corpusculo quodam adiposo in hominum genis obvio. Diss. inang. Dorpat 1853). Dieser Körper entwickelt sich zuweilen zu einer lipomatösen Geschwulst, die sich dann in der Wange bald mehr nach vorn, bald mehr nach hinten hervordrängt, und, wie man aus der Zusammenstellung, die Bruns (V. v. Bruns, Handbuch der practischen Chirurgie. Abth. II. Bd. I. S. 146, 1134. Vgl. Gant, The Lancet. 1856. Vol. II. No. 23) über diese Fälle geliefert hat, ersehen kann, nicht selten Veranlassung gegeben hat, sie mit Parotisgeschwülsten zu verwechseln. Indem das Gewächs von hinten her die Parotis hervorschiebt, so ist es manchmal nicht wohl möglich, die Drüse davon zu unterscheiden. Sehr leicht kann man daher eine solche Bildung als eine Parotisgeschwulst behandeln, und bei der Exstirpation die Parotis selbst unnöthigerweise mit entfernen."

Bruns stellt eine kleine Anzahl genauer untersuchter Fettgeschwülste in den Wangen zusammen, welche sich zum Theil, nachdem wir jetzt die Structur des Saugpolsters kennen gelernt haben, mit Sicherheit als aus demselben entstanden zu erkennen geben. 
Beispielsweise führe ich aus der Beschreibung eines von Auvert mitgetheilten Falles folgende Stelle an ${ }^{1}$ ): „die ausgeschnittene Geschwulst von Gansei-Grösse und gelapptem Ansehen ward von einem blättrigen Żellgewebe wie von einer Hülle eingeschlossen, welche zugleich Fortsetzungen in das Innere der Masse hinein entsendete und dieselbe dadurch in vielfache Fücher absonderte, die mit weicher kugeliger Fettmasse gefüllt waren".

In der Beschreibung eines anderen Falles von Albino-Magri (bei Bérard, Maladies de la glande parotide) ${ }^{2}$ ) heisst es: "Bei einem Längenschnitte durch den vorderen Theil des Lipoms fand man dasselbe von zwei Membranen eingeschlossen, einer äusseren, dicken, ligamentösen, der Dura mater ähnlichen Haut, welche sich leicht von der inneren, dünnen, zellgewebigen Haut abziehen liess, von welcher Fortsätze nach innen sich erstreckten, die die Geschwulstmasse in Lappen and diese wieder in Läppchen absonderten. Die in diesen Lappen enthaltene Masse war von verschiedener Beschaffenheit, der bei weitem grösste Theil derselben war Fett" .....

Wie gerechtfertigt Virchow's Bemerkung war, dass der Fettkörper der Wange immer wieder vergessen werde, zeigt ein Blick auf die nach 1863 veröffentlichten Lehrbücher der Anatomie.

J. Henle, Handbuch der Eingeweidelehre des Menschen, Braunschweig 1866, S. 133 erwähnt nur „einen Fettklumpen, der die unter dem Jochbogen befindliche Lücke ausfüllt." In demselben Handbuch finden sich bildliche Darstellungen von zwei Horizontalschnitten des Kopfes durch die Commissur der Lippen, bei welchen offenbar das Polster getroffen wurde, und zwar an einem Fötusschädel S. 82 Fig. 46 und dem Kopf eines Erwachsenen S. 145 Fig. 105. In beiden Abbildungen ist das Wangenfett als eine gleichartige Fettmasse dargestellt.

Auch Hubert Luschka beschränkt sich auf Allgemeinheiten. Er sagt in seiner Anatomie des menschlichen Kopfes, Tübingen 1867, S. 308: "So lange der zwischen dem Masseter und Buccinatorius befindliche Zwischenraum - Cavitas buccalis — wie bei wohlgenährten Menschen, von Fett erfüllt ist, bietet die Backe eine volle Rundung dar, während dieselbe durch den

i) Bruns, a. a. O. S. 149.

2) Derselbe S. 1135 . 


\section{4}

Schwund des Fettes bis zu dem Maasse grubenartig einsinken kann, dass sich der vordere Rand des Masseter deutlich abhebt," und auf S. 310 ,das subcutane Gewebe schliesst eine wechselnde, die Abrundung der Backe bedingende, Menge Fett ein, mit dessen Schwund dieselbe daher einfällt, oder sich in Falten legt."

In Quain's Lehrbuch der Anatomie, bearbeitet von Karl Ernst Emil Hoffmann, Erlangen 1870, findet sich eine kurze Erwähnung des Fettgewebes der Wange nur bei der Beschreibung des Verlaufs des Duct. Stenonianus auf S. 448.

N. Rüdinger, Topographisch-chirurgische Anatomie des Menschen, III. Abtheilung, Stuttgart 1874, giebt Folgendes an (S. 66 u. f.): "Das subcutane Fettgewebe zeigt sich von ungleicher Dicke. Bei hochgradiger Ablagerung des Fettes entsteht die Fülle und Rundung des Gesichtes, wie man sie vorwiegend bei Kindern und Frauen findet. Schwindet das Fett bei ungenügender Ernährung, bei Krankheit oder im vorgerückten Alter, so entsteht jenes leere eingefallene Gesicht, in welchem die Bewegungen der mimischen und Kau-Muskeln viel auffallender erscheinen, als bei Personen mit starker Fettablagerung ..... Während das subcutane Fettlager auf der Ohrspeicheldrüse und dem M. masseter mässig stark auftritt, erreicht es in der Regio infraorbitalis and der R. buccalis unter dem Wangenbein die grösste Mächtigkeit".. und S. 70: "die Aussenseite des Buccinator ist von einem stärkeren Fettlager erfüllt, als die des Masseter. Von dem Grade der Ablagerung dieses Fettes ist die Fülle oder Leere der Backengegend abhängig. Bei Schwụnd des Fettes sinkt am auffallendsten die Gegend ron dem Masseter und unter dem Wangenbeine ein."

Von deutschen Autoren ist noch Prof. Dr. Carl Langer zu erwähnen, welcher in seiner Anatomie der äusseren Formen des menschlichen Körpers, Wien 1884, auf S. 17 sich folgendermaassen äussert: Am reichlichsten ist das Fett beim Kinde in der Backengegend angehäuft, deren geradezu kugelige Wölhung von einem losen Fettklampen herrührt, welcher sich, unter dem Kaumuskel hervorwuchernd, auf den Backenmuskel lagert" und auf S. 155: "Obgleich die Niveaudifferenz zwischen diesen beiden Muskeln (Masseter und Buccinator) eine ansehnliche ist, so gehen doch bei einigem Fettansatze beide Regionen ohne Grenze in- 
einander über; es buchtet und rundet sich sogar die Auflage auf den Backenmuskel, wenn sich ausser der gewöhnlichen Fettunterlage der Haut auch jener besondere, unter dem Unterkieferaste hervorwuchernde Fettklumpen vorfindet, welcher an gutgenährten Neugebornen immer vorhanden ist."

Den Schluss dieser historischen Skizze möge wieder ein französischer Schriftsteller machen, bei welchem wir im Anschluss an Bichat und Malgaigne bisher die genauesten Angaben über die Topographie des Polsters beim Erwachsenen finden, während er zugleich anch werthvolle Winke giebt über pathologische Beziehangen desselben zu den Lipomen der Backengegend. Es ist dies der bereits mehrfach genannte französische Anatom P. Tillaux ${ }^{1}$. Derselbe schreibt: „Entre le muscle buccinateur et son aponévrose, on rencontre le groupe des glandes molaires reposant directement sur le muscle: ces glandes sont situées en arrière du canal du Sténon et correspondent aux deux dernières grosses molaires. Il n'est pas très rare de trouver, dans l'epaisseur des joues, des Kystes et des adénomes qui ont leur origine dans ces glandes. En arrière de ce groupe glandulaire, et sur le même plan, ce trouve une boule graisseuse constante, même chez les sujets les plus émaciés, appelée bonle de Bichat. Elle répond au bord antérieur de la branche montante, au bord antérieur et un peu à la face interne du masséter, quelle separe du buccinateur.

Ia joue présente donc deux couches de graisse distinctes: l'une superficielle sous-cutanée, sous-aponévrotique; l'autre profonde, sous-aponévrotique. La première couche communique avec le tissu cellulo-adjpeux sous-cutané des regions temporale et parotidienne; la seconde se continue avec les graisses de la fosse zygomatique, par son intermédiaire avec la couche sous-aponévrotique de la region temporale, et aussi avec le tissu celluloadipeux làche occupant le fond de la loge parotidienne."

Tillaux zeigt dann, welchen Weg Eitersenkungen in der Backe auf Grund der angegebenen Verhältnisse nehmen müssen und fährt auf p. 290 fort: "Les détails anatomiques dans lesquels je viens d'entrer jettent une certaine clarté sur la question

1) a. a. 0. S. 289 .

Archiv f. pathol. Anat. Ba. XCVII. Hft. 3. 
encore obscure dos lipomes de la joue. Ces tumeurs se développent aux dépens des deux couches de tissu adipeux qui existent dans la région; ils sont done: les uns sous-cutanés ou sous-aponévrotiques. C'est à tort qu'on designe ces derniers sous le nom de sous-muqueux, car ils ne siégent jamais dïrectement sous la muqueuse, mais bien à la face externe du muscle buccinateur; la cause de l'erreur vient de ce que bridés en dehors par l'aponévrose, ces derniers font saillie dans la bouche plutôt que sous la peau. On concoit qu'un lipome sous-aponévrotique de la joue puisse gagner la fosse zygomatique, la fosse temporale, et forme une tumeur profonde d'ont le diagnostic sera fort difficile. On concoit également que des lipomes développés dans Ie même point se propagent à la loge parotidienne en passant derrière la branche montante, ou que réciproquement ils viennent de la région parotidienne faire saillie dans la joue.

Cette étiologie des lipomes géniens offre plus qu'un intérêt théorique. En effet, si le lipome est sous-aponévrotique, on devra l'attaquer par la muqueuse buccale" .......

Damit dürften die wesentlichsten auf das Saugpolster zu beziehenden Angaben in der Literatur erschöpft sein.

\section{Erklärung der Abbildungen.}

Tafel XVIII - XIX.

Fig. 1. Fünf Monate alter atrophischer Knabe, an dessen Regio buccalis das Saugpolster deutlich hervortritt. Durch das Weinen während der photographischen Momentaufnahme markirt sich das Polster besonders deutlich.

Fig. 2. Darstellung des Saugpolsters eines Neugeborenen von aussen. 1 Masseter. 2 Musc. temporalis. 3 Parotis mit ihrem Ausführungsgang. 4 Subcutaneus colli mit dem Risorius Santorini zurückgeschlagen. 5 Saugpolster, welches den M. buccinator fast ganz und den M'. masseter theilweise deckt. 6 Stiel des Saugpolsters.

Fig. 3. Darstellang des Saugpolsters mit seinem Stiel in der Regio retromaxillaris bis zur Fossa sphenopalatina hinauf. 1 Jochbogen, berausgesägt und mit dem daranhängenden Masseter nach abwärts geschlagen. 2 Aufsteigender Ast des Unterkiefers. 3 Os zygomaticum. 4 Aeste des Unterkiefers mit dem 5 Musc. temporalis nach aufwärts umgebogen. 6 Parotis. 7 Musc. buccinator. 8 Abgerundeter unterer 


\section{7}

Theil des Saugpolsters, etwas stark gedehnt, dawit 9 der Stiel bis zur Fossa sphenopalatina sichtbar wurde.

Fig. 4. Horizontalschnitt durch die Backen und die Mundböhle eines Neugebornen, wobei das Saugpolster annähernd in seinem grössten Umfange getroffen ist. 1 Schlundkopf. 2 Das hintere Ende des grossen Zungenbeinhorns. 3 Musc. pharyngopalatinus. 4 Dorsum lingnae. 5. Kehldeckel mit dem Introitus laryngis. 6 Aufsteigender Ast des Unterkiefers. 7 MIusc. pterygoideus internus. 8 Mrusc. masseter. 9 Mruse. buccinator. 10 Yinse. orbicularis oris. 11 Subcutaneus colli und Risorius Santorini. 12 Saugpolster unmittelbar an die äzssere Fläche des Musc. buccinator angrenzend.

Fig. 5: Horizontalschnitt durch das blauinjicirte und rothimbibirte Saugpolster eines Neugebornen mit der Backenschleimhaut und dem MIusc. buccinator, unter sechsfacher Vergrösserung. I Backenschleimhaut. 2 Stark entwickelter Buccinator quer durchschnitten. 3 Verbindungsstelle des Saugpolsters, mit dem Busc. buccinator. 4 Kapsel ${ }^{\circ}$ des Saugpolsters. 5. Fettgrippen in Innern des Polsters durch Bindegewebszüge getrennt. 7 Besondere laterale Fettgruppe. 8 u. 9 Angrenzende Bindesubstanz mit Fettpartien, welche ein von dem Saugpolster abweichendes Verhalten zeigen.

Fig. 6. Ein Stück des blauinjicirten und rothimbibirten Saugpolsterrandes, bei $81 / 1$ Vergrösserung gezeichuet. 1 Bindegewebskapsel des Saugpolsters, welche sich 2 in der Nähe der Fettzellen sebr dicht zeigt. 3 u. 4 Bindegewebsbalken im. Innern des PoIsters, welche das Fett in einzelne Gruppen abgrenzen. 5 u. 6 Fettzellen mit injicirten Capillaren. 7 Grosse Gefässstämme an der Aussenseite des Saugpolsters.

Fig. 7. Frontalschnitt des Kopfes eines etwa 4 Monate alten Fötus, unter 7facher Vergrösserung gezeichnet. Der Schnitt ist aus. einer Collection von frontalen Durchschaitten des Kopfes ausgewählt. I Unterkiefer. 2 Frontalschnitt der Zunge. 3 Yruse. buccinator stark entwickelt. 4 u. 5 Die oberfächliche Muskellage, welche theils von dem Ricorius Santorini, theils von dem Subcutaneus colli abstammt, liegt dem Saugpolster unmittelbar an und deckt dasselbe von aussen. 6 Saugpolster, weiches dem Musc. buccinator nicht nnmittelbar anliegt, aber doch wit demselben dureh einen breiten Faserzing in Verbindung steht. 

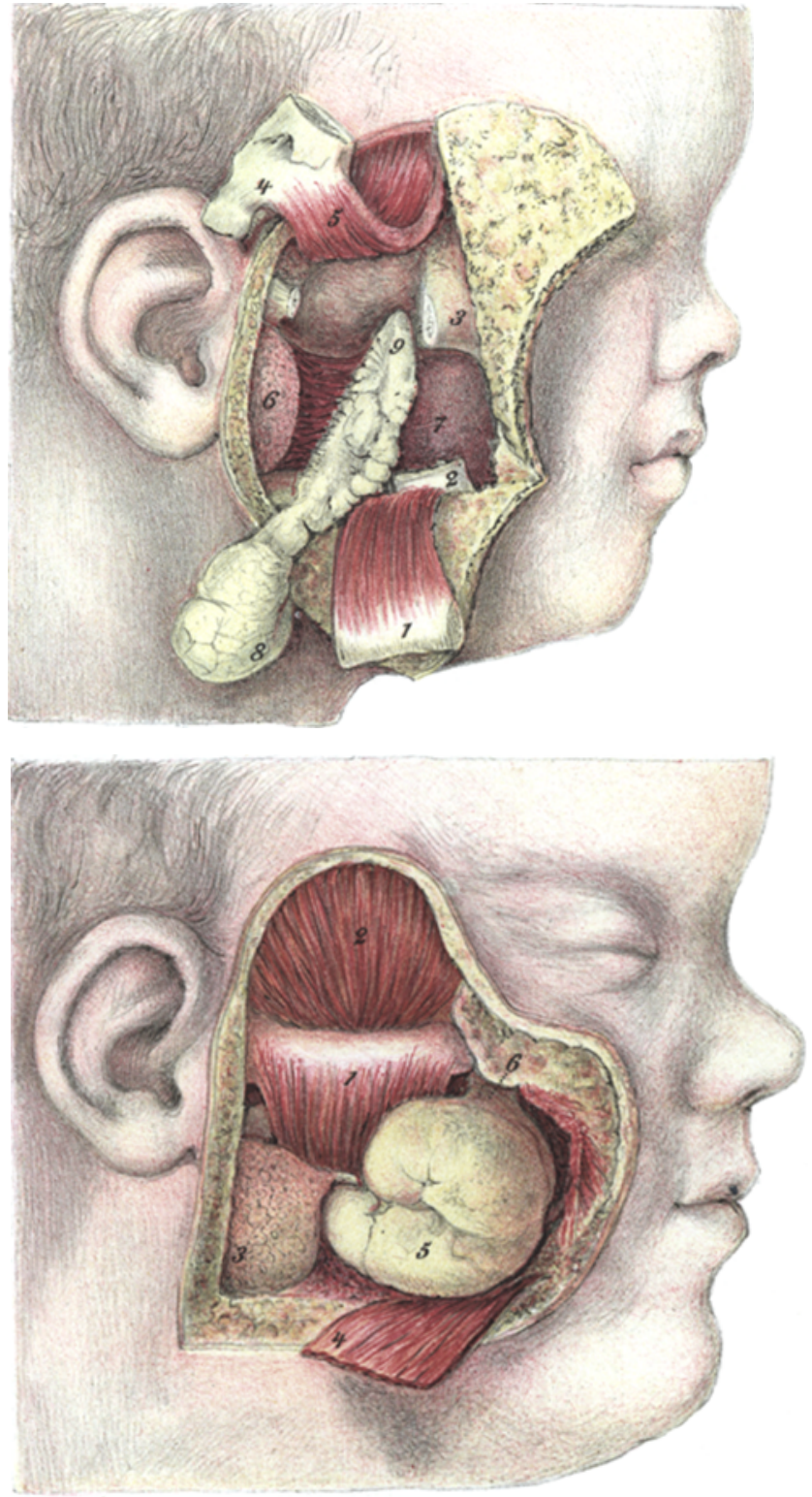

$\%$ 


\section{5. $6 / 7$}
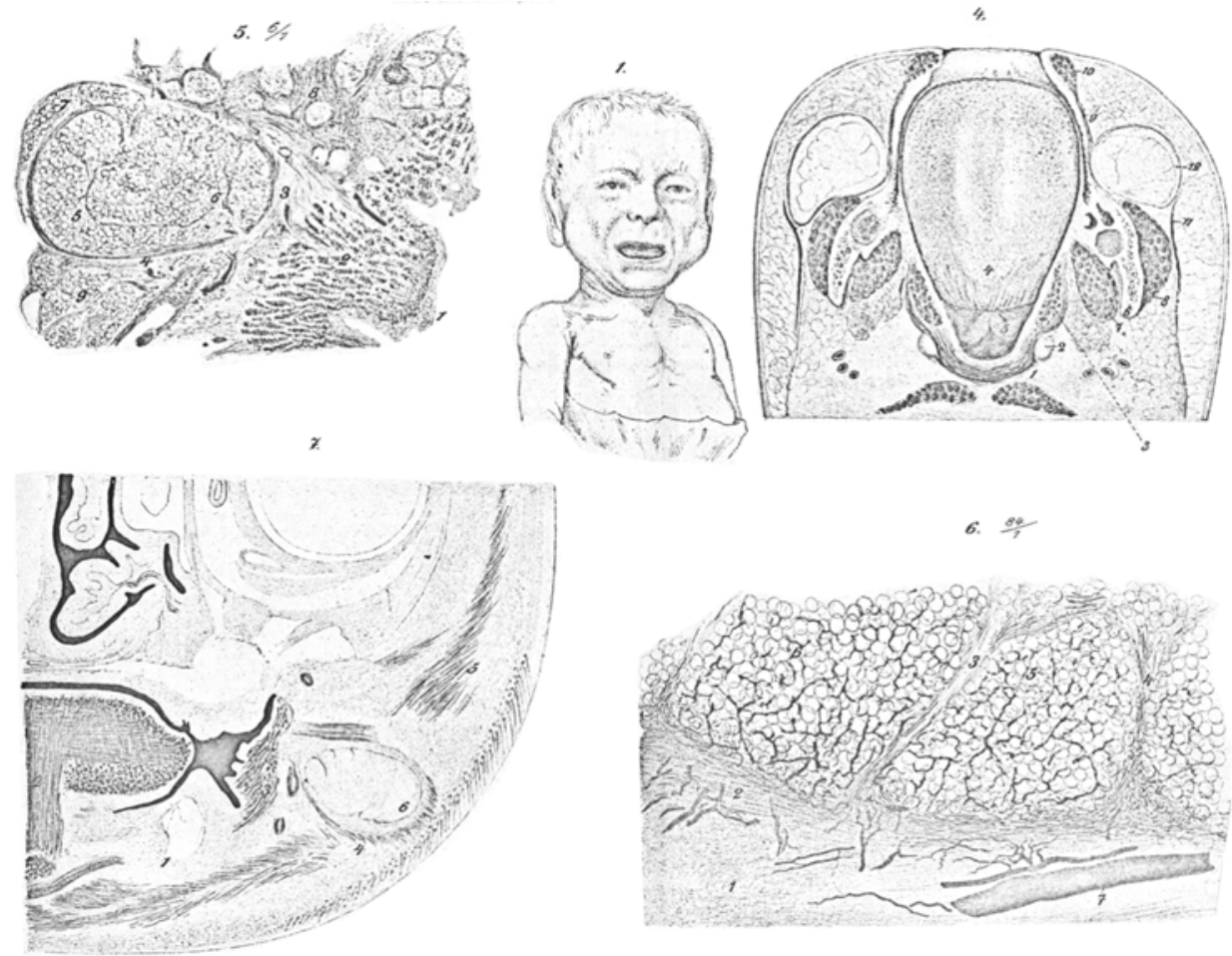

Twart Fint 